\title{
Limit analysis of the statistics of quasi-steady non-linear aerodynamic forces for small turbulence intensities
}

\author{
Vincent Denoël
}

January 20, 2009

\author{
University of Liège, Department of Architecture, Geology, Environment and Constructions, \\ Structural Engineering Division \\ Chemin des Chevreuils, 1, Bât B52/3, 4000 Liège, Belgium. \\ email: v.denoel@ulg.ac.be
}

Tel: +32 (0) 43662930 // Fax: +32 (0) 43669192.

\begin{abstract}
This paper focuses on the estimation of statistical characteristics of a specific quasisteady wind loading used in buffeting analyses. In this loading, the intrinsic non-linearity of aerodynamic coefficients is considered and approached by a polynomial expression of any a priori chosen degree. As rigorous developments of the statistical moments would result in impractical formulations, we suggest to consider the smallness of the turbulence intensities to construct, by means of a limit analysis, approximate expressions of the raw moments of aerodynamic forces. From these expressions, approximate cumulants and associated dimensionless characteristics, such as skewness and excess coefficients, are derived. The accuracy of the proposed analytical relations is assessed by comparison with Monte Carlo simulations, and the relevance of the sophisticated non-linear loading under consideration is compared to more traditional models.
\end{abstract}

Turbulence, non-linear, aerodynamic coefficient, limit analysis, correlation, wind intensity, bridge deck.

\section{Introduction}

Buffeting analyses play an important role in the design of civil engineering structures subjected to the action of wind. This kind of analysis consists in assessing the dynamic response of a structure to a random aerodynamic loading.

In the atmospheric boundary layer, the wind velocity exhibits a turbulent, and hence random, character. In 2-D applications, it is commonly modeled as a mean wind velocity $U$ and two zero-mean turbulence components $u$ and $w$ (e.g. [1]), that are written in a dimensionless way in the present approach. Figure 1 schematically represents the wind forces on a bridge deck, in laminar (a) and turbulent (b) flows. The general developments of this paper are equivalently valid for any of these three forces (drag, lift, moment). Recent researches have 
shown a slight non-Gaussianity of the turbulence components $u$ and $w$ [2], in medium to high altitudes. Nevertheless, at lower altitudes relevant to bridge engineering applications, the turbulence field $(u, v)$ is usually modeled as a 2-variable Gaussian process (e.g. [1, 3]). Whenever the importance of the project justifies it, on-site measurements are performed and a 2-variable Gaussian process is fitted to the measured data. On the other hand, for minor applications, codes and standards (e.g. [4]) might be consulted for indications about the selection of the turbulence parameters (mainly the standard deviations of $u$ and $w$ ).

Because of the 2-D nature of the turbulence, the wind incidence $\alpha$ instantly changes (Fig. 1 ). It is expressed as a non-linear function of $u$ and $w$, by means of geometric relations, and is therefore a non-Gaussian random process. Convenient expressions of its first four statistical moments are given in [5].

Several wind loading models exist [6] to express aerodynamic forces as a function of the relative wind velocities and incidence. Among them, the quasi-steady model provides a satisfactory representation for low wind velocities (compared to structural velocities). As a particular case of Navier-Stokes equations, it states that an aerodynamic force is proportional to the squared velocity $V^{2}$ and to the aerodynamic coefficient $c(\alpha)$, which is itself expressed as a function of the wind incidence $\alpha$. A dimensionless expression of this force is:

$$
f=c(\alpha)\left[(1+u)^{2}+w^{2}\right] .
$$

The first applications of statistical concepts to buffeting analyses seem to be due to Liepmann [7]. In these seminal developments, the wind velocity field was however limited to one turbulence component $u$, resulting therefore in constant wind incidence and aerodynamic coefficient $\left(c(\alpha)=c_{0}\right)$. Also, for the sake of simplicity, the aerodynamic loading was linearized with respect to $u$ in order to provide a set of Gaussian aerodynamic forces, $f=c_{0}(1+2 u)$.

A first upgrade of this theory consists in avoiding the linearization of the loading, and therefore in considering in (1) terms proportional to the squared velocity, but still in a 1$\mathrm{D}$ wind field, with a constant aerodynamic coefficient, $f=c_{0}(1+u)^{2}$. This more formal approach, adopted by $[8,9,10,11]$ among others, results in a non-Gaussian loading, which consequently brings more complexity in the structural analysis. For moderate wind intensities, Benfratello et al [10] have shown a non negligible deviation of the statistical characteristics (of the loading) from those obtained with a Gaussian model.

In this paper, we present another enhancement of the model. We consider the same nonlinearity as that described before, but extend the developments to a 2-D turbulence field, including therefore a more rigorous expression of the force as given in (1). First, a quadratic term related to the second turbulence component $w$ is considered. As expected, this generates another slight non-Gaussianity, but of the same order of magnitude as the one indicated by $[8,9,10,11]$. Secondly, the introduction of the second component of the turbulence causes the wind incidence to continuously vary in time. Hence, the variation of the aerodynamic coefficients with respect to the wind incidence $\alpha$ has to be considered (Fig. 2). As these are noticeable non-linear functions and because the wind incidence itself is already non-Gaussian, this is a second origin of the non-Gaussianity of the loading. With realistic turbulence intensities and aerodynamic coefficients, seminal developments [12] revealed that the non-linearity of the aerodynamic coefficients is actually the main source of non-Gaussianity of the loading. Hereafter the investigations related to this model are further pursued.

Figure 2 depicts examples of aerodynamic coefficients, resulting from wind-tunnel measurements. As already mentioned, they are non-linear; in practical applications, their erratic 


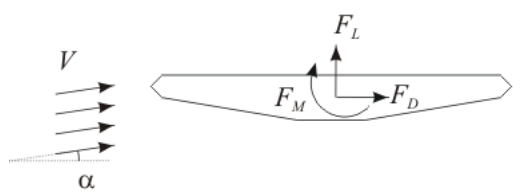

(a)

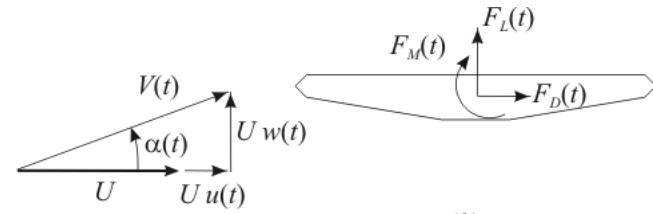

(b)

Figure 1: Wind forces induced on a bridge deck in (a) laminar and (b) turbulent flows. In a quasi-steady model, the evolution of forces in a turbulent flow is adapted from the laminar case, excepted that the variation with time $t$ is considered.

character is usually smoothed by fitting a polynomial to the measured data. Several polynomial representations are discussed in [5]. Aiming at a general development, this paper treats such a polynomial approximation, of any a priori given degree. The best linear and cubic fits according to the stochastic linearization principle, see [5], are represented in Fig. 2, as well as the linear fit obtained from the cubic fit by setting coefficients of higher degree terms to zero. As illustrated in Section 7, this way of linearizing the aerodynamic coefficient provides an accurate estimate of the coefficient, but in a narrow range of wind incidences around zero, and is therefore limited to small turbulence intensities.

With this wind model based on (i) a quadratic expression of the force, (ii) a non-linear geometric expression of the wind incidence, and (iii) a polynomial approximation of the aerodynamic coefficients, exact developments of the statistical moments of the forces would result in unusable formulations. However, among the multitude of terms of these formal formulations, only a couple contribute significantly to the expression of the moments. The difference of order between the different terms is attributable to the smallness of the turbulence intensities. For this reason, a limit analysis for small turbulence intensities is considered in order to extract these significant terms. Next developments are performed for the purpose of providing the same level of accuracy, regardless of the relative importance of the coefficients of the polynomial approximation.

\section{Statistics of the turbulence field}

The joint probability density function of the dimensionless components of the turbulence $u$ and $w$ is usually assumed to be Gaussian (e.g. $[1,3]$ )

$$
p_{u w}(u, w)=\frac{1}{2 \pi I_{u} I_{w} \sqrt{1-\rho^{2}}} e^{\frac{-1}{2\left(1-\rho^{2}\right)}\left(\frac{u^{2}}{I_{u}^{2}}-\frac{2 \rho u w}{I_{u} I_{w}}+\frac{w^{2}}{I_{w}^{2}}\right)}
$$

where $\rho, I_{u}$ and $I_{w}$ represent the correlation coefficient and the turbulence intensities. In this paper, it is supposed that both turbulence components are small and have similar orders of magnitude

$$
I_{u}=\iota_{u} \varepsilon \quad ; \quad I_{w}=\iota_{w} \varepsilon
$$

where $\iota_{u}=o(1), \iota_{w}=o(1)$ and $\varepsilon \ll 1$ is a small parameter $(\varepsilon \simeq 0.1$ in typical applications). This assumption is usually met; it is major in view of the limit analysis for small $\varepsilon$ that is next developed. The $(l ; n-l)$ joint raw moment of the turbulence components is defined as

$$
\mu_{u^{l} w^{n-l}}=E\left[u^{l} w^{n-l}\right]=\iint_{-\infty}^{+\infty} u^{l} w^{n-l} p_{u w}(u, w) d u d w
$$



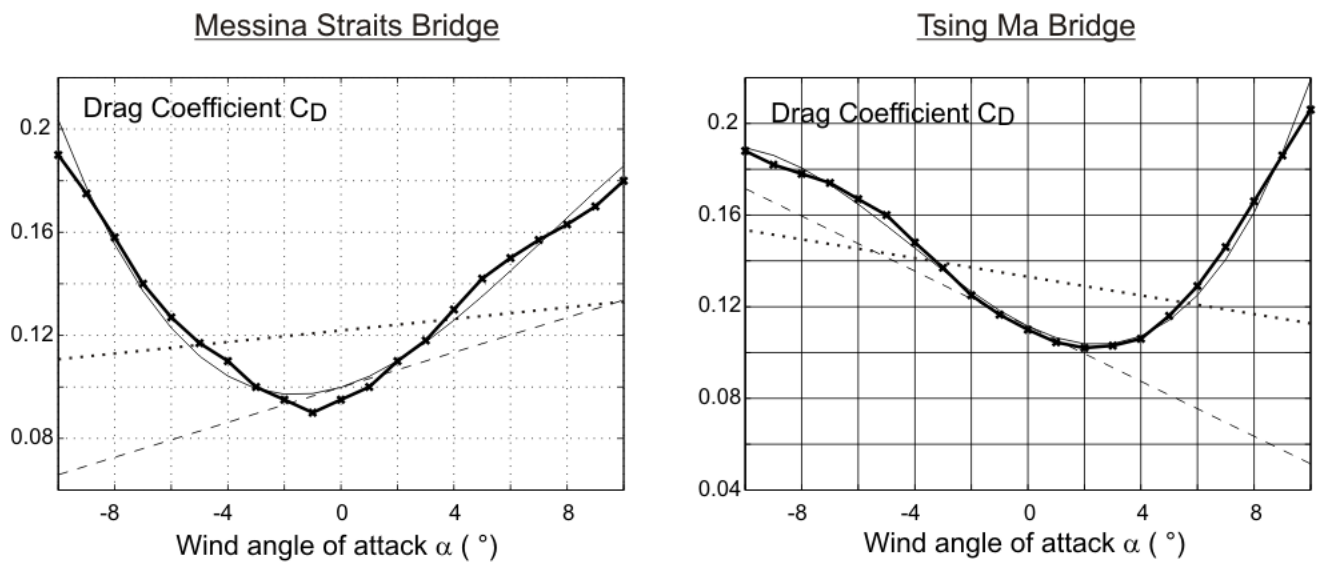

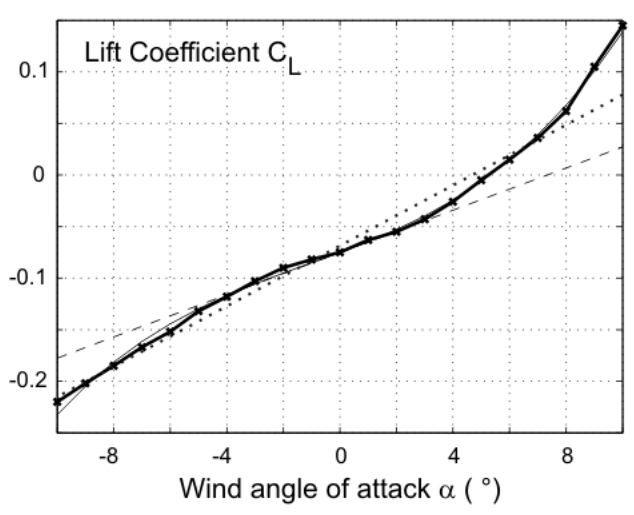

(a)

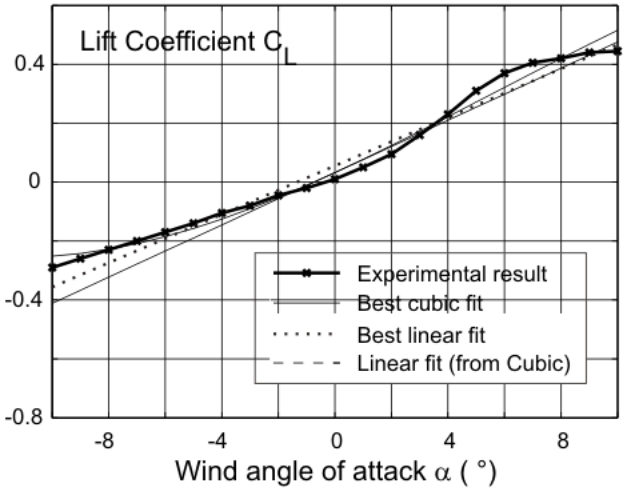

(b)

Figure 2: Examples of aerodynamic coefficients, resulting from wind-tunnel measurements. Adapted from [13, 14]. Best cubic fits and best linear fits are obtained by applying the method proposed in [5]. Also linear fits obtained by neglecting coefficients of higher degree terms in cubic fit are represented by dashed lines. 
The introduction of a binomial development for $u^{l}$ as

$$
u^{l}=\iota_{u}^{l}\left(\frac{u}{\iota_{u}}-\frac{\rho w}{\iota_{w}}+\frac{\rho w}{\iota_{w}}\right)^{l}=\iota_{u}^{l} \sum_{t=0}^{l}\left(\begin{array}{c}
l \\
t
\end{array}\right)\left(\frac{u}{\iota_{u}}-\frac{\rho w}{\iota_{w}}\right)^{t}\left(\frac{\rho w}{\iota_{w}}\right)^{l-t}
$$

allows a separation of the double integral. After some changes of variables and simplifications, the raw moment is expressed by means of Gaussian integrals

$$
\mu_{u^{l} w^{n-l}}=\frac{\varepsilon^{n} \iota_{u}^{l} \iota_{w}^{n-l}}{2} \sum_{t=0}^{l}\left(\begin{array}{c}
l \\
t
\end{array}\right)\left(\sqrt{1-\rho^{2}}\right)^{t} \rho^{l-t} \frac{1}{\sqrt{\pi}} \int_{-\infty}^{+\infty} p^{t} e^{\frac{-p^{2}}{2}} d p \frac{1}{\sqrt{\pi}} \int_{-\infty}^{+\infty} q^{n-t} e^{\frac{-q^{2}}{2}} d w
$$

and a closed form expression of the moment is finally obtained

$$
\mu_{u^{l} w^{n-l}}=\varepsilon^{n} \iota_{u}^{l} \iota_{w}^{n-l} \begin{cases}\sum_{t=0}^{\lfloor l / 2\rfloor}\left(\begin{array}{c}
l \\
2 t
\end{array}\right)\left(1-\rho^{2}\right)^{t} \rho^{l-2 t}(2 t-1) ! !(n-2 t-1) ! ! & \text { for } n \text { even } \\
0 & \text { for } n \text { odd }\end{cases}
$$

where the symbol $\lfloor x\rfloor$ represents the integer part of $x$, and the symbol !! denotes the double factorial

$$
n ! != \begin{cases}n(n-2)(n-4) \ldots 3.1 & \text { for } n>0, \text { odd } \\ n(n-2)(n-4) \ldots 4.2 & \text { for } n>0, \text { even } \\ 1 & \text { for } n \leq 0 .\end{cases}
$$

As particular cases, for $n$ even, it is possible to write (7) for the first few values of $l$

$$
\begin{aligned}
\mu_{w^{n}} & =\varepsilon^{n} \iota_{w}^{n}(n-1) ! ! \\
\mu_{u w^{n-1}} & =\varepsilon^{n} \iota_{u} \iota_{w}^{n-1} \rho(n-1) ! ! \\
\mu_{u^{2} w^{n-2}} & =\varepsilon^{n} \iota_{u}^{2} \iota_{w}^{n-2}\left(1+(n-2) \rho^{2}\right)(n-3) ! ! \\
\mu_{u^{3} w^{n-3}} & =\varepsilon^{n} \iota_{u}^{3} \iota_{w}^{n-3} \rho\left(3+(n-4) \rho^{2}\right)(n-3) ! ! \\
\mu_{u^{4} w^{n-4}} & =\varepsilon^{n} \iota_{u}^{4} \iota_{w}^{n-4}\left[3+(n-4)\left(6+(n-6) \rho^{2}\right) \rho^{2}\right](n-5) ! ! \\
\mu_{u^{5} w^{n-5}} & =\varepsilon^{n} \iota_{u}^{5} \iota_{w}^{n-5} \rho\left[15+(n-6)\left(10+(n-8) \rho^{2}\right) \rho^{2}\right](n-5) ! !
\end{aligned}
$$

\section{$3 \quad$ Wind loading model}

The dimensionless aerodynamic force is expressed as the product of the non-linear aerodynamic coefficient and the squared wind velocity [12]:

$$
f=c(\alpha)\left[(1+u)^{2}+w^{2}\right]
$$

where the angle of attack is expressed, from geometrical relations (Fig. 1), as

$$
\alpha=\arctan \frac{w}{1+u} .
$$

In this model, the aerodynamic coefficient is represented by a polynomial of an a priori chosen degree $N$ as 


$$
c(\alpha)=\sum_{i=0}^{N} \frac{c_{i}}{i !} \alpha^{i} .
$$

For the simplicity of the forthcoming relations, the aerodynamic force (10) is written

$$
f=\sum_{i=0}^{N} \phi_{i} c_{i}
$$

where

$$
\phi_{i}(u, w)=\frac{1}{i !}\left[\arctan \frac{w}{1+u}\right]^{i}\left[(1+u)^{2}+w^{2}\right] .
$$

As $u$ and $w$ are $o(\varepsilon) \ll 1$, we introduce a Taylor series expansion of $\phi_{i}(u, w)$ in the vicinity of $(u, v)=(0,0)$, followed by a change of variables introducing the total order of derivation $n$

$$
\phi_{i}=\sum_{n=0}^{\infty} \sum_{l=0}^{n} \delta_{n, l, i} u^{l} w^{n-l}
$$

where

$$
\delta_{n, l, i}=\frac{1}{n !}\left(\begin{array}{c}
n \\
l
\end{array}\right)\left(\frac{\partial^{n} \phi_{i}(u, w)}{\partial u^{l} \partial w^{n-l}}\right)_{(u, w)=(0,0)} .
$$

Figure 3 collects the values of $\delta_{n, l, i}$ for the first few values of $i$. An explicit expression could be obtained for $\delta_{n, l, i}$, as a solution of a recurrence. This goes however beyond the scope of this paper, and would anyway result in tedious developments and an explicit expression that is so complex that its use would be unusable. The best definition of coefficients $\delta_{n, l, i}$ remains therefore (16). From this definition, it is possible to prove that

$$
\delta_{n, l, i}=0 \quad \text { for } n<i+l \vee(n+l+i) \bmod 2=1
$$

and that the first non-zero values of $\delta_{n, l, i}$ take these forms

$$
\begin{aligned}
& \delta_{i, 0, i}=\frac{1}{i !} \quad ; \quad \delta_{i+1,1, i}=\frac{2-i}{i !} \\
& \delta_{i+2,0, i}=\frac{3-i}{3 i !} \quad ; \quad \delta_{i+3,1, i}=\frac{-i(3-i)}{3 i !} \\
& \delta_{i+2,2, i}=\frac{(1-i)(2-i)}{2 i !} \quad ; \quad \delta_{i+3,3, i}=\frac{-i(1-i)(2-i)}{6 i !} \\
& \delta_{i+4,0, i}=\frac{(5 i-17) i}{90 i !} ; \quad \delta_{i+4,2, i}=\frac{i(i+1)(3-i)}{6 i !} \\
& \delta_{i+4,4, i}=\frac{i(i+1)(1-i)(2-i)}{24 i !} ; \quad \delta_{i+5,1, i}=\frac{\left(-5 i^{2}+7 i+34\right) i}{90 i !} \\
& \delta_{i+5,3, i}=\frac{-(i+1)(i+2)(3-i) i}{18 i !} ; \quad \delta_{i+5,5, i}=\frac{-(i+1)(i+2) i(1-i)(2-i)}{120 i !} .
\end{aligned}
$$




\begin{tabular}{|c|c|c|c|c|c|c|}
\hline$\delta_{n, l, 0}$ & $l=0$ & $l=1$ & $l=2$ & $l=3$ & $l=4$ & $l=5$ \\
\hline$n=0$ & 1 & -7 & - & & & \\
\hline$n=1$ & 0 & 2 & 7 & & & \\
\hline$n=2$ & 1 & 0 & 1 & & & \\
\hline$n=3$ & 0 & 0 & 0 & 0 & & \\
\hline$n=4$ & 0 & 0 & 0 & 0 & 0 & \\
\hline$n=5$ & 0 & 0 & 0 & 0 & 0 & 0 \\
\hline$n=6$ & 0 & 0 & 0 & 0 & 0 & 0 \\
\hline$n=7$ & 0 & 0 & 0 & 0 & 0 & 0 \\
\hline$n=8$ & 0 & 0 & 0 & 0 & 0 & 0 \\
\hline
\end{tabular}

\begin{tabular}{|c|c|c|c|c|c|c|}
\hline$\delta_{n, l, 1}$ & $l=0$ & $l=1$ & $l=2$ & $l=3$ & $l=4$ & $l=5$ \\
\hline$n=0$ & 0 & $A$ & & & & \\
\hline$n=1$ & 1 & 0 & 7 & & & \\
\hline$n=2$ & 0 & 1 & 0 & 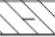 & & \\
\hline$n=3$ & $2 / 3$ & 0 & 0 & 0 & -1 & \\
\hline$n=4$ & 0 & $-2 / 3$ & 0 & 0 & 0 & \\
\hline$n=5$ & $-2 / 15$ & 0 & $2 / 3$ & 0 & 0 & 0 \\
\hline$n=6$ & 0 & $2 / 5$ & 0 & $-2 / 3$ & 0 & 0 \\
\hline$n=7$ & $2 / 35$ & 0 & $-4 / 5$ & 0 & $2 / 3$ & 0 \\
\hline$n=8$ & 0 & $-2 / 7$ & 0 & $4 / 3$ & 0 & $-2 / 3$ \\
\hline
\end{tabular}

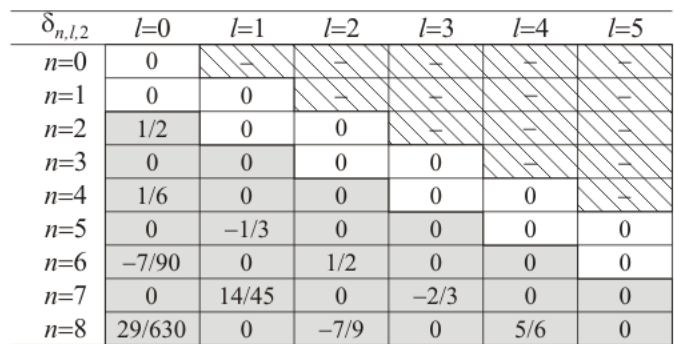

\begin{tabular}{|c|c|c|c|c|c|c|}
\hline$\delta_{n, l, 3}$ & $l=0$ & $l=1$ & $l=2$ & $l=3$ & $l=4$ & $l=5$ \\
\hline$n=0$ & 0 & $\rightarrow$ & 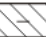 & & & \\
\hline$n=1$ & 0 & 0 & 7 & & & \\
\hline$n=2$ & 0 & 0 & 0 & - & & \\
\hline$n=3$ & $1 / 6$ & 0 & 0 & 0 & & \\
\hline$n=4$ & 0 & $-1 / 6$ & 0 & 0 & 0 & \\
\hline$n=5$ & 0 & 0 & $1 / 6$ & 0 & 0 & 0 \\
\hline$n=6$ & 0 & 0 & 0 & $-1 / 6$ & 0 & 0 \\
\hline$n=7$ & $-1 / 90$ & 0 & 0 & 0 & $1 / 6$ & 0 \\
\hline$n=8$ & 0 & $1 / 18$ & 0 & 0 & 0 & $-1 / 6$ \\
\hline
\end{tabular}

\begin{tabular}{|c|c|c|c|c|c|c|}
\hline$\delta_{n, l, 4}$ & $l=0$ & $l=1$ & $l=2$ & $l=3$ & $l=4$ & $l=5$ \\
\hline$n=0$ & 0 & 8 & & & & \\
\hline$n=1$ & 0 & 0 & $\Phi$ & $t$ & & \\
\hline$n=2$ & 0 & 0 & 0 & + & & \\
\hline$n=3$ & 0 & 0 & 0 & 0 & & \\
\hline$n=4$ & $1 / 24$ & 0 & 0 & 0 & 0 & I \\
\hline$n=5$ & 0 & $-1 / 12$ & 0 & 0 & 0 & 0 \\
\hline$n=6$ & $-1 / 72$ & 0 & $1 / 8$ & 0 & 0 & 0 \\
\hline$n=7$ & 0 & $1 / 18$ & 0 & $-1 / 6$ & 0 & 0 \\
\hline$n=8$ & $1 / 180$ & 0 & $-5 / 36$ & 0 & $5 / 24$ & 0 \\
\hline
\end{tabular}

\begin{tabular}{|c|c|c|c|c|c|c|}
\hline$\delta_{n, l, 5}$ & $l=0$ & $l=1$ & $l=2$ & $l=3$ & $l=4$ & $l=5$ \\
\hline$n=0$ & 0 & 17 & 71 & & & \\
\hline$n=1$ & 0 & 0 & (1) & & & \\
\hline$n=2$ & 0 & 0 & 0 & 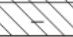 & 5 & \\
\hline$n=3$ & 0 & 0 & 0 & 0 & $\Delta$ & \\
\hline$n=4$ & 0 & 0 & 0 & 0 & 0 & -1 \\
\hline$n=5$ & $1 / 120$ & 0 & 0 & 0 & 0 & 0 \\
\hline$n=6$ & 0 & $-1 / 40$ & 0 & 0 & 0 & 0 \\
\hline$n=7$ & $-1 / 180$ & 0 & $1 / 20$ & 0 & 0 & 0 \\
\hline$n=8$ & 0 & $1 / 36$ & 0 & $-1 / 12$ & 0 & 0 \\
\hline
\end{tabular}

Figure 3: Coefficients $\delta_{n, l, i}$ for $i=0,1, \ldots 5$. 
From a practical viewpoint, as $u$ and $w$ are $o(\varepsilon) \ll 1$, the first few non-zero terms in $(15)$ might be kept, and an approximation of (15) writes

$$
\begin{aligned}
\phi_{i}= & a_{i} w^{i}\left[1+b_{i} u+\left(c_{i} w^{2}+c_{i}^{\prime} u^{2}\right)+\left(d_{i} u w^{2}+d_{i}^{\prime} u^{3}\right)\right. \\
& +\left(e_{i} w^{4}+e_{i}^{\prime} u^{2} w^{2}+e_{i}^{\prime \prime} u^{4}\right) \\
& \left.+\left(f_{i} u w^{4}+f_{i}^{\prime} u^{3} w^{2}+f_{i}^{\prime \prime} u^{5}\right)+\ldots\right]
\end{aligned}
$$

where

$$
\begin{aligned}
& a_{i}=\delta_{i, 0, i} \quad ; \quad b_{i}=\frac{\delta_{i+1,1, i}}{a_{i}} \quad ; \quad c_{i}=\frac{\delta_{i+2,0, i}}{a_{i}} \\
& c_{i}^{\prime}=\frac{\delta_{i+2,2, i}}{a_{i}} \quad ; \quad d_{i}=\frac{\delta_{i+3,1, i}}{a_{i}} \quad ; \quad d_{i}^{\prime}=\frac{\delta_{i+3,3, i}}{a_{i}} \\
& e_{i}=\frac{\delta_{i+4,0, i}}{a_{i}} \quad ; \quad e_{i}^{\prime}=\frac{\delta_{i+4,2, i}}{a_{i}} \quad ; \quad e_{i}^{\prime \prime}=\frac{\delta_{i+4,4, i}}{a_{i}} \\
& f_{i}=\frac{\delta_{i+5,1, i}}{a_{i}} \quad ; \quad f_{i}^{\prime}=\frac{\delta_{i+5,3, i}}{a_{i}} \quad ; \quad f_{i}^{\prime \prime}=\frac{\delta_{i+5,5, i}}{a_{i}}
\end{aligned}
$$

are introduced for the compacity of the following developments.

\section{Mean aerodynamic force}

A general expression of the mean force is obtained by substituting (15) into (13) and introducing the mathematical expectation operator $E[]$

$$
\mu_{f}=E[f]=\sum_{i=0}^{N} \bar{\phi}_{i} c_{i}
$$

where $\bar{\phi}_{i}=E\left[\phi_{i}(u, w)\right]$. The introduction of (15) and (16) into (22) provides an expression of the mean force in terms of $\varepsilon, \iota_{u}, \iota_{w}$ and $\rho$. For $N=2$, i.e. a quadratic aerodynamic coefficient, and a truncation of the summation on $n$ to $n=6$, the exhaustive development of this expression yields

$$
\begin{aligned}
\mu_{f}= & c_{0}+\left(c_{0} I_{u}^{2}+c_{0} I_{w}^{2}+\frac{c_{2} I_{w}^{2}}{2}+c_{1} I_{u} I_{w} \rho\right)+\frac{c_{2} I_{w}^{4}}{2}-2 c_{1} I_{u} I_{w}^{3} \rho \\
& -\frac{c_{3} I_{u} I_{w}^{3} \rho}{2}+\frac{3 c_{2} I_{u}^{2} I_{w}^{4}}{2}-\frac{7 c_{2} I_{w}^{6}}{6}-6 c_{1} I_{u}^{3} I_{w}^{3} \rho-\frac{3 c_{3} I_{u}^{3} I_{w}^{3} \rho}{2} \\
& +6 c_{1} I_{u} I_{w}^{5} \rho+6 c_{2} I_{u}^{2} I_{w}^{4} \rho^{2}-4 c_{1} I_{u}^{3} I_{w}^{3} \rho^{3}-c_{3} I_{u}^{3} I_{w}^{3} \rho^{3} .
\end{aligned}
$$

This expression of the mean force is heavy and similar developments for higher statistical moments would evidently provide much more complex formulations. Nevertheless these expressions may be significantly simplified, by considering that the wind intensity is small. The relative orders of magnitude of coefficients $c_{i}$, however, are a priori unknown as it is desired to keep general developments regarding the shape of the aerodynamic coefficient. The statistical moments are therefore expressed by grouping terms with respect to $c_{i}$ 's, as it is 
done in (22). The limit analysis is then performed separately on each of their coefficients $\bar{\phi}_{i}$, which are expressed as

$$
\bar{\phi}_{i}=E\left[\phi_{i}(u, w)\right]=\sum_{\substack{n=0 \\ n \text { even }}}^{\infty} \sum_{l=0}^{n} \delta_{n, l, i} \mu_{u^{l} w^{n-l}}
$$

where the restriction to even values of $n$ results from (7).

After, we construct an approximation of $\bar{\phi}_{i}$ as the sum of a leading order term $\bar{\phi}_{i, o}$ and the first two corrections $\bar{\phi}_{i, 1}$ and $\bar{\phi}_{i, 2}$. As $\mu_{u^{l} w^{n-l}}$ is of order $\varepsilon^{n}$, the leading order term is obtained by truncating the summation on $n$ to $n^{*}$, the smallest value of $n$ for which there exists at least one value of $l$, with $0 \leq l \leq n$, such that $\delta_{n, l, i}$ is non zero. In view of properties (17), the double summation reduces to a single term corresponding to $(n, l)=(i, 0)$ for $i$ even, and $(n, l)=(i+1,1)$ for $i$ odd

$$
\bar{\phi}_{i, o}= \begin{cases}\delta_{i, 0, i} \mu_{w^{i}} & \text { for } i \text { even } \\ \delta_{i+1,1, i} \mu_{u w^{i}} & \text { for } i \text { odd }\end{cases}
$$

which is, in addition, confirmed by the explicit developments (20).

The first correction $\bar{\phi}_{i, 1}$ corresponds to the next value of $n$ strictly superior to $n^{*}$, such that $\delta_{n, l, i}$ is non zero. In view of properties $(17)$, two terms have to be kept for this first correction. They correspond to $(n, l)=(i+2,0)$ and $(n, l)=(i+2,2)$ for $i$ even, and $(n, l)=(i+3,1)$ and $(n, l)=(i+3,3)$ for $i$ odd

$$
\bar{\phi}_{i, 1}= \begin{cases}\delta_{i+2,0, i} \mu_{w^{i+2}}+\delta_{i+2,2, i} \mu_{u^{2} w^{i}} & \text { for } i \text { even } \\ \delta_{i+3,1, i} \mu_{u w^{i+2}}+\delta_{i+3,3, i} \mu_{u^{3} w^{i}} & \text { for } i \text { odd. }\end{cases}
$$

Similarly, the second correction $\bar{\phi}_{i, 2}$ is

$$
\bar{\phi}_{i, 2}= \begin{cases}\delta_{i+4,0, i} \mu_{w^{i+4}}+\delta_{i+4,2, i} \mu_{u^{2} w^{i+2}}+\delta_{i+4,4, i} \mu_{u^{4} w^{i}} & \text { for } i \text { even } \\ \delta_{i+5,1, i} \mu_{u w^{i+4}}+\delta_{i+5,3, i} \mu_{u^{3} w^{i+2}}+\delta_{i+5,5, i} \mu_{u^{5} w^{i}} & \text { for } i \text { odd }\end{cases}
$$

Substitution of (9) and (18) into (25), (26) and (27) yields the expected approximation of $\bar{\phi}_{i}=\bar{\phi}_{i, o}+\bar{\phi}_{i, 1}+\bar{\phi}_{i, 2}$, including the first two corrections. Each term is written as

$$
\begin{aligned}
\bar{\phi}_{i, o}= & \frac{1}{i ! !} \varepsilon^{i} \iota_{w}^{i} \\
\bar{\phi}_{i, 1}= & \frac{1}{i ! !} \varepsilon^{i+2} \iota_{w}^{i}\left[\frac{(i+1)(3-i)}{3} \iota_{w}^{2}+\frac{(1-i)(2-i)}{2}\left(1+\rho^{2} i\right) \iota_{u}^{2}\right] \\
\bar{\phi}_{i, 2}= & \frac{(i-1) ! !}{i !} \varepsilon^{i+4} \iota_{w}^{i}\left[\frac{(5 i-17)(i+1)(i+3) i}{90} \iota_{w}^{4}+\frac{i(i+1)^{2}(3-i)}{6}\left(1+(i+2) \rho^{2}\right) \iota_{u}^{2} \iota_{w}^{2}\right. \\
& \left.\quad+\frac{i(i+1)(3-i)}{6}\left[3+i\left(6+(i-2) \rho^{2}\right) \rho^{2}\right] \iota_{u}^{4}\right]
\end{aligned}
$$


for $i$ even, and

$$
\begin{aligned}
\bar{\phi}_{i, o}= & \frac{\varepsilon^{i+1} \iota_{u} \iota_{w}^{i} \rho}{(i-1) ! !}(2-i) \\
\bar{\phi}_{i, 1}= & \frac{-\varepsilon^{i+3} \iota_{u} \iota_{w}^{i} \rho}{(i-1) ! !}\left[\frac{i(i+2)(3-i)}{3} \iota_{w}^{2}+\frac{i(1-i)(2-i)}{6}\left(3+\rho^{2}(i-1)\right) \iota_{u}^{2}\right] \\
\bar{\phi}_{i, 2}= & \frac{i ! !}{i !} \varepsilon^{i+5} \iota_{u} \iota_{w}^{i} \rho\left[\frac{\left(-5 i^{2}+7 i+34\right)(i+2)(i+4) i}{90} \iota_{w}^{4}\right. \\
& +\frac{-(i+1)(i+2)^{2}(3-i) i}{18}\left(3+(i+1) \rho^{2}\right) \iota_{u}^{2} \iota_{w}^{2} \\
& \left.+\frac{-(i+1)(i+2) i(1-i)(2-i)}{120}\left[15+(i-1)\left(10+(i-3) \rho^{2}\right) \rho^{2}\right] \iota_{u}^{4}\right]
\end{aligned}
$$

for $i$ odd. As an example, for $N=2$, i.e. a quadratic aerodynamic coefficient, the approximate mean force (with the first correction only) writes

$$
\mu_{f} \simeq c_{0}\left(1+I_{u}^{2}+I_{w}^{2}\right)+I_{u} I_{w} \rho c_{1}\left(1-2 I_{w}^{2}\right)+\frac{I_{w}^{2}}{2} c_{2}\left(1+I_{w}^{2}\right)
$$

where the physical wind intensities $I_{u}$ and $I_{w}$, see (3), are reintroduced. This expression of the mean aerodynamic force is evidently much simpler than (23) and presents the advantage of offering the same level of approximation for each coefficient $c_{i}$.

It is interesting to notice that the first correction is actually a second order correction, and that the second correction is actually a fourth order one. Hence, nothing justifies so far the need to include the second correction, and even probably the first one either. This need will however become clear in light of the following developments.

\section{Raw moments of aerodynamic force}

Developments similar to those presented in section 4 are applied to construct approximations of raw moments. The $m^{\text {th }}$ raw moment of the aerodynamic force is defined as

$$
\mu_{f^{m}}=E\left[f^{m}\right]=E\left[\left(\sum_{i=0}^{N} \phi_{i} c_{i}\right)^{m}\right]=\sum_{i_{1}=0}^{N} \ldots \sum_{i_{m}=0}^{N} E\left[\prod_{p=1}^{m} \phi_{i_{p}}\right]\left(\prod_{p=1}^{m} c_{i_{p}}\right) .
$$

Again, as no particular assumption is formulated concerning aerodynamic coefficients, the approximate solution is obtained by a limit analysis of the coefficients of the combinations of $c_{i}$ 's. The limit analysis is therefore performed on

$$
\Phi^{(m)}=E\left[\prod_{p=1}^{m} \phi_{i_{p}}\right]
$$

Similarly to the developments related to the mean force, this infinite series -because of the presence of $\phi_{i_{p}}$, see (15)- might be truncated to its first terms, and the coefficient $\Phi^{(m)}$ is written as the sum of a leading order term and successive corrections:

$$
\Phi^{(m)}=\Phi_{o}^{(m)}+\Phi_{1}^{(m)}+\Phi_{2}^{(m)}+\ldots
$$


Let us introduce these functions of the set of indices $i_{1}, \ldots, i_{p}$

$$
\mathcal{P}=\frac{1}{\prod_{p=1}^{m} a_{i_{p}}}=i_{1} ! \ldots i_{p} ! \quad \text { and } \quad \mathcal{S}_{k}=\sum_{p=1}^{m} i_{p}^{k} .
$$

Substitution of (20) for each factor of the product in (32) yields

$$
\begin{aligned}
& \prod_{p=1}^{m} \phi_{i_{p}}=\frac{w^{\mathcal{S}_{1}}}{\mathcal{P}} \prod_{p=1}^{m} {\left[1+b_{i_{p}} u+\left(c_{i_{p}} w^{2}+c_{i_{p}}^{\prime} u^{2}\right)+\left(d_{i_{p}} u w^{2}+d_{i_{p}}^{\prime} u^{3}\right)+\ldots\right.} \\
&\left.+\left(e_{i_{p}} w^{4}+e_{i_{p}}^{\prime} u^{2} w^{2}+e_{i_{p}}^{\prime \prime} u^{4}\right)+\left(f_{i_{p}} u w^{4}+f_{i_{p}}^{\prime} u^{3} w^{2}+f_{i_{p}}^{\prime \prime} u^{5}\right)\right] .
\end{aligned}
$$

where the product on $p$ is then extended, and where only the lowest powers of $u$ and $w$ are kept

$$
\begin{aligned}
\prod_{p=1}^{m} \phi_{i_{p}}= & \frac{w^{\mathcal{S}_{1}}}{\mathcal{P}}\left[1+\alpha_{1} u+\left(\alpha_{2} w^{2}+\alpha_{2}^{\prime} u^{2}\right)+\left(\alpha_{3} u w^{2}+\alpha_{3}^{\prime} u^{3}\right)\right. \\
& \left.+\left(\alpha_{4} w^{4}+\alpha_{4}^{\prime} u^{2} w^{2}+\alpha_{4}^{\prime \prime} u^{4}\right)+\left(\alpha_{5} u w^{4}+\alpha_{5}^{\prime} u^{3} w^{2}+\alpha_{5}^{\prime \prime} u^{5}\right)\right] .
\end{aligned}
$$

Functions $\alpha_{i}\left(i_{1}, \ldots, i_{p}\right)$ are obtained by comparison of likewise powers of $u^{k} w^{l}$ in (35) and (36). They are expressed with basic algebraic functions. The first ones are straightforwardly obtained as

$$
\begin{aligned}
\alpha_{1} & =\sum_{p=1}^{m} b_{i_{p}}=\sum_{p=1}^{m} 2-i_{p}=2 \mathcal{S}_{0}-\mathcal{S}_{1} \\
\alpha_{2} & =\sum_{p=1}^{m} c_{i_{p}}=\sum_{p=1}^{m} \frac{3-i_{p}}{3}=\mathcal{S}_{0}-\frac{1}{3} \mathcal{S}_{1} \\
\alpha_{2}^{\prime} & =\sum_{p=1}^{m} c_{i_{p}}^{\prime}+\sum_{p=1}^{m-1} \sum_{q=p+1}^{m} b_{i_{p}} b_{i_{q}} \\
& =\frac{1}{2}\left(-1+2 \mathcal{S}_{0}-\mathcal{S}_{1}\right)\left(2 \mathcal{S}_{0}-\mathcal{S}_{1}\right)
\end{aligned}
$$

whereas similar but longer developments are required for the following ones. After simpli- 
fications, one can obtain:

$$
\begin{aligned}
\alpha_{3}= & \frac{1}{3}\left(-2+2 \mathcal{S}_{0}-\mathcal{S}_{1}\right)\left(3 \mathcal{S}_{0}-\mathcal{S}_{1}\right) \\
\alpha_{3}^{\prime}= & \frac{1}{6}\left(-2+2 \mathcal{S}_{0}-\mathcal{S}_{1}\right)\left(-1+2 \mathcal{S}_{0}-\mathcal{S}_{1}\right)\left(2 \mathcal{S}_{0}-\mathcal{S}_{1}\right) \\
\alpha_{4}= & \frac{1}{90}\left(13 \mathcal{S}_{1}+5\left(9\left(-1+\mathcal{S}_{0}\right) \mathcal{S}_{0}-6 \mathcal{S}_{0} \mathcal{S}_{1}+\mathcal{S}_{1}^{2}\right)\right) \\
\alpha_{4}^{\prime}= & \frac{1}{6}\left(-3+2 \mathcal{S}_{0}-\mathcal{S}_{1}\right)\left(-2+2 \mathcal{S}_{0}-\mathcal{S}_{1}\right)\left(3 \mathcal{S}_{0}-\mathcal{S}_{1}\right) \\
\alpha_{4}^{\prime \prime}= & \frac{1}{24}\left(-3+2 \mathcal{S}_{0}-\mathcal{S}_{1}\right)\left(-2+2 \mathcal{S}_{0}-\mathcal{S}_{1}\right)\left(-1+2 \mathcal{S}_{0}-\mathcal{S}_{1}\right)\left(2 \mathcal{S}_{0}-\mathcal{S}_{1}\right) \\
\alpha_{5}= & \frac{1}{90}\left(-4+2 \mathcal{S}_{0}-\mathcal{S}_{1}\right)\left(13 \mathcal{S}_{1}+5\left(9\left(-1+\mathcal{S}_{0}\right) \mathcal{S}_{0}-6 \mathcal{S}_{0} \mathcal{S}_{1}+\mathcal{S}_{1}^{2}\right)\right) \\
\alpha_{5}^{\prime}= & \frac{1}{18}\left(-4+2 \mathcal{S}_{0}-\mathcal{S}_{1}\right)\left(-3+2 \mathcal{S}_{0}-\mathcal{S}_{1}\right)\left(-2+2 \mathcal{S}_{0}-\mathcal{S}_{1}\right)\left(3 \mathcal{S}_{0}-\mathcal{S}_{1}\right) \\
\alpha_{5}^{\prime \prime}= & \frac{1}{24}\left[32 \mathcal{S}_{0}^{4}+6 \mathcal{S}_{1}^{4}+\mathcal{S}_{1}^{3}\left(89-2 \mathcal{S}_{2}\right)-8 \mathcal{S}_{0}^{3}\left(21+12 \mathcal{S}_{1}-2 \mathcal{S}_{2}\right)+4 \mathcal{S}_{2}\left(6 \mathcal{S}_{2}-\mathcal{S}_{3}\right)\right. \\
& +\mathcal{S}_{1}^{2}\left(374-48 \mathcal{S}_{2}+4 \mathcal{S}_{3}\right)+4 \mathcal{S}_{0}^{2}\left(70+\mathcal{S}_{1}\left(119+24 \mathcal{S}_{1}-6 \mathcal{S}_{2}\right)-36 \mathcal{S}_{2}+4 \mathcal{S}_{3}\right) \\
& +\mathcal{S}_{1}\left(3\left(-96+\mathcal{S}_{2}\right) \mathcal{S}_{2}+64 \mathcal{S}_{3}-6 \mathcal{S}_{4}\right)-2 \mathcal{S}_{0}\left(72+20 \mathcal{S}_{1}^{3}+\mathcal{S}_{1}^{2}\left(187-6 \mathcal{S}_{2}\right)\right) \\
& \left.-2 \mathcal{S}_{0}\left(\mathcal{S}_{2}\left(-208+3 \mathcal{S}_{2}\right)+56 \mathcal{S}_{3}+\mathcal{S}_{1}\left(380-84 \mathcal{S}_{2}+8 \mathcal{S}_{3}\right)-6 \mathcal{S}_{4}\right)\right] \\
& +\frac{79 \mathcal{S}_{1}-10\left(8 \mathcal{S}_{2}-4 \mathcal{S}_{3}+\mathcal{S}_{4}\right)+\mathcal{S}_{5}}{5} .
\end{aligned}
$$

The mathematical expectation of (36) ultimately provides the approximate coefficients $\Phi^{(m)}$. Since the even joint moments of the components of the turbulence are equal to zero, see (7), terms in (36) may again be collected by two following orders, as it was done for the mean force.

The leading order and the first two corrections are therefore expressed as

$$
\begin{aligned}
\Phi_{o}^{(m)}= & \frac{1}{\mathcal{P}} \mu_{w^{\mathcal{S}_{1}}}=\frac{\left(\mathcal{S}_{1}-1\right) ! !}{\mathcal{P}} \varepsilon^{\mathcal{S}_{1}} \iota_{w} \mathcal{S}_{1} \\
\Phi_{1}^{(m)}= & \frac{\alpha_{2}}{\mathcal{P}} \mu_{w^{\mathcal{S}_{1}+2}}+\frac{\alpha_{2}^{\prime}}{\mathcal{P}} \mu_{u^{2} w^{\mathcal{S}_{1}}} \\
= & \frac{\left(\mathcal{S}_{1}-1\right) ! !}{\mathcal{P}}\left[\alpha_{2}\left(\mathcal{S}_{1}+1\right) \iota_{w}^{2}+\alpha_{2}^{\prime}\left(\rho^{2} \mathcal{S}_{1}+1\right) \iota_{u}^{2}\right] \varepsilon^{\mathcal{S}_{1}+2} \iota_{w}^{\mathcal{S}_{1}} \\
\Phi_{2}^{(m)}= & \frac{\alpha_{4}}{\mathcal{P}} \mu_{w^{\mathcal{S}_{1}+4}}+\frac{\alpha_{4}^{\prime}}{\mathcal{P}} \mu_{u^{2} w^{\mathcal{S}_{1}+2}}+\frac{\alpha_{4}^{\prime \prime}}{\mathcal{P}} \mu_{u^{4} w^{\mathcal{S}_{1}}} \\
= & \frac{\left(\mathcal{S}_{1}-1\right) ! !}{\mathcal{P}}\left[\alpha_{4}\left(\mathcal{S}_{1}+3\right)\left(\mathcal{S}_{1}+1\right) \iota_{w}^{4}+\alpha_{4}^{\prime}\left(1+\left(\mathcal{S}_{1}+2\right) \rho^{2}\right)\left(\mathcal{S}_{1}+1\right) \iota_{u}^{2} \iota_{w}^{2}\right. \\
& \left.\quad+\alpha_{4}^{\prime \prime}\left[3+\mathcal{S}_{1}\left(6+\left(\mathcal{S}_{1}-2\right) \rho^{2}\right) \rho^{2}\right] \iota_{u}^{4}\right] \mathcal{S}^{\mathcal{S}_{1}+4} \iota_{w}^{\mathcal{S}_{1}}
\end{aligned}
$$


for $\mathcal{S}_{1}$ even, and

$$
\begin{aligned}
\Phi_{o}^{(m)}= & \frac{\alpha_{1}}{\mathcal{P}} \mu_{u w} \mathcal{S}_{1}=\frac{\mathcal{S}_{1} ! ! \rho}{\mathcal{P}} \alpha_{1} \varepsilon^{\mathcal{S}_{1}+1} \iota_{u} \iota_{w}^{\mathcal{S}_{1}} \\
\Phi_{1}^{(m)}= & \frac{\alpha_{3}}{\mathcal{P}} \mu_{u w^{\mathcal{S}_{1}+2}}+\frac{\alpha_{3}^{\prime}}{\mathcal{P}} \mu_{u^{3} w^{\mathcal{S}_{1}}} \\
= & \frac{\mathcal{S}_{1} ! ! \rho}{\mathcal{P}}\left[\alpha_{3}\left(\mathcal{S}_{1}+2\right) \iota_{w}^{2}+\alpha_{3}^{\prime}\left(\rho^{2} \mathcal{S}_{1}+3-\rho^{2}\right) \iota_{u}^{2}\right] \varepsilon^{\mathcal{S}_{1}+3} \iota_{u} \iota_{w}^{\mathcal{S}_{1}} \\
\Phi_{2}^{(m)}= & \frac{\alpha_{5}}{\mathcal{P}} \mu_{u w} \mathcal{S}_{1}+4+\frac{\alpha_{5}^{\prime}}{\mathcal{P}} \mu_{u^{3} w^{\mathcal{S}_{1}+2}}+\frac{\alpha_{5}^{\prime \prime}}{\mathcal{P}} \mu_{u^{5} w^{\mathcal{S}_{1}}} \\
= & \frac{\mathcal{S}_{1} ! ! \rho}{\mathcal{P}}\left[\alpha_{5}\left(\mathcal{S}_{1}+4\right)\left(\mathcal{S}_{1}+2\right) \iota_{w}^{4}+\alpha_{5}^{\prime}\left(3+\left(\mathcal{S}_{1}+1\right) \rho^{2}\right)\left(\mathcal{S}_{1}+2\right) \iota_{u}^{2} \iota_{w}^{2}\right. \\
& \left.\quad+\alpha_{5}^{\prime \prime}\left[15+\left(\mathcal{S}_{1}-1\right)\left(10+\left(\mathcal{S}_{1}-3\right) \rho^{2}\right) \rho^{2}\right] \iota_{u}^{4}\right] \varepsilon^{\mathcal{S}_{1}+5} \iota_{u} \iota_{w}
\end{aligned}
$$

for $\mathcal{S}_{1}$ odd.

The approximate expression (31) of the raw moment of the aerodynamic force finally writes

$$
\mu_{f^{m}}=E\left[f^{m}\right]=\sum_{i_{1}=0}^{N} \ldots \sum_{i_{m}=0}^{N}\left(\Phi_{o}^{(m)}+\Phi_{1}^{(m)}+\Phi_{2}^{(m)}\right)\left(\prod_{p=1}^{m} c_{i_{p}}\right) .
$$

By way of example, for $N=2$, the first approximate moments (without the second correction) are

$$
\begin{aligned}
\mu_{f^{2}} \simeq & c_{0}^{2}\left(1+6 I_{u}^{2}+2 I_{w}^{2}\right)+c_{1}^{2} I_{w}^{2}\left(1+\left(1+2 \rho^{2}\right) I_{u}^{2}+4 I_{w}^{2}\right)+c_{0} c_{2} I_{w}^{2}\left(1+\left(1+2 \rho^{2}\right) I_{u}^{2}+4 I_{w}^{2}\right) \\
+ & c_{2}^{2} I_{w}^{4}\left(\frac{3}{4}+\frac{5 I_{w}^{2}}{2}\right)+c_{0} c_{1} I_{u} I_{w} \rho\left(6+6 I_{u}^{2}+10 I_{w}^{2}\right)+c_{1} c_{2} I_{u} I_{w}^{3} \rho\left(3-15 I_{w}^{2}\right) \\
\mu_{f^{3}} \simeq & c_{0}^{3}\left(1+15 I_{u}^{2}+3 I_{w}^{2}\right)+c_{1}^{3} \rho I_{u} I_{w}^{3}\left(9+3\left(3+2 \rho^{2}\right) I_{u}^{2}+30 I_{w}^{2}\right)+c_{2}^{3} \frac{15 I_{w}^{6}}{8}\left(1+7 I_{w}^{8}\right) \\
& +c_{0}^{2} c_{1} I_{u} I_{w} \rho\left(15+90 I_{u}^{2}+72 I_{w}^{2}\right)+c_{0}^{2} c_{2} I_{w}^{2}\left(\frac{3 I_{w}^{2}}{2}+\left(1+2 \rho^{2}\right) 9 I_{u}^{2}+\frac{21 I_{w}^{2}}{2}\right) \\
& +c_{1}^{2} c_{2} I_{w}^{4}\left(\frac{9}{2}+\frac{9}{2}\left(1+4 \rho^{2}\right) I_{u}^{2}+\frac{75 I_{w}^{2}}{2}\right)+3 c_{0} c_{1}^{2} I_{w}^{2}\left(1+6\left(1+2 \rho^{2}\right) I_{u}^{2}+7 I_{w}^{2}\right) \\
& +c_{0} c_{2}^{2} I_{w}^{4}\left(\frac{9}{4}+\frac{9}{4}\left(1+4 \rho^{2}\right) I_{u}^{2}+\frac{75 I_{w}^{2}}{4}\right)+c_{1} c_{2}^{2} I_{u} I_{w}^{5} \rho\left(\frac{45}{4}-105 I_{w}^{2}\right) \\
& +c_{0} c_{1} c_{2} I_{u} I_{w}^{3} \rho\left(27+9\left(3+2 \rho^{2}\right) I_{u}^{2}+90 I_{w}^{2}\right) .
\end{aligned}
$$

\section{Variance, skewness and excess of aerodynamic force}

The physical interpretation of statistical properties is usually made easier by consideration of standard deviation and dimensionless quantities, such as skewness and excess coefficients [15]. These values are derived from the cumulants $\kappa_{f}$ of the aerodynamic force, whose estimation is the reason for the need to develop above two corrections in addition to the leading order. Indeed, as a convincing argument, let us suppose for a moment that the aerodynamic coefficient 
is constant, and that the first correction only is considered. The expression of the approximate mean force (30) reduces therefore to

$$
\mu_{f}=c_{0}\left(\bar{\phi}_{i, o}+\bar{\phi}_{i, 1}\right)=c_{0}\left(1+\varepsilon^{2} \iota_{u}^{2}+\varepsilon^{2} \iota_{w}^{2}\right)
$$

which is actually exact, as seen from the mathematical expectation of (10). The second moment (44) reduces to

$$
\mu_{f^{2}}=c_{0}^{2}\left(\Phi_{o}^{(2)}+\Phi_{1}^{(2)}+O\left(\varepsilon^{4}\right)\right)=c_{0}^{2}\left[1+2 \varepsilon^{2}\left(3 \iota_{u}^{2}+\iota_{w}^{2}\right)+O\left(\varepsilon^{4}\right)\right] .
$$

In the resulting approximate expression of the second cumulant

$$
\kappa_{f^{2}}=\mu_{f^{2}}-\mu_{f}^{2}=c_{0}^{2}\left(4 \varepsilon^{2} \iota_{u}^{2}+O\left(\varepsilon^{4}\right)\right)
$$

the coefficients of terms $c_{0}^{2} O(0)$ have canceled, and the error is now two orders below the leading one, whereas it was four orders below for the raw moment. Pushing the reasoning further again, the consideration of the leading term only in the approximate expressions of the raw moments, would result in an unacceptable discrepancy $\left(\kappa_{f^{2}}=0\right)$.

As a matter of fact, this particular case justifies the need to include at least the first correction in the expression of the raw moments, and eventually the second one if sufficient precision has to be achieved on the estimation of cumulants. Also, any discrepancy on the cumulants is straightforwardly reported on the quantities of interest in this study, i.e. the standard deviation and the skewness and excess coefficients, as these are expressed as

$$
\sigma_{f}=\sqrt{\kappa_{f^{2}}} \quad ; \quad \gamma_{3, f}=\frac{\kappa_{f^{3}}}{\sigma_{f}^{3}} \quad ; \quad \gamma_{e, f}=\frac{\kappa_{f^{4}}}{\sigma_{f}^{4}} .
$$

\section{Applications}

Next we assess the accuracy of the proposed relations and compare the results provided by the complete non-linear quasi-steady wind loading with more traditional models. To this aim, drag and lift coefficients of the Messina Straits Bridge project and Tsing Ma Bridge are considered. The best linear and cubic fit obtained for these coefficients, shown in Fig. 2, are characterized by coefficients $c_{i}$ given in Table 1 . A third fit is also considered as the linear fit obtained with the first two coefficients of the cubic model. The difference between both linear fits is evident from the graphical representation of Fig. 2, and from values given in Table 1.

Both drag coefficients evince a significant cubic component, whereas lift coefficients show a somewhat more linear character, for which a complex non-linear model is probably not required. This statement is illustrated in Section 7.2.

\subsection{Validation of the proposed relations}

The accuracy of the proposed approximate relations is assessed by comparison with Monte Carlo simulations. Series of $1,000,000$ samples of the turbulence components are first generated, for various correlation coefficients $(\rho=0, \rho=-0.5$ and $\rho=-1)$ covering the range of variation expected in practical applications [16]. Also, the longitudinal turbulence intensity $I_{u}$ is assumed to vary between 0 and 0.15 , whereas the transverse turbulence intensity is chosen as $I_{w}=I_{u} / 2$. As instantaneous statistics are considered in the subject matter, no particular frequency content has to be imposed for the simulations. The numerical simulation 

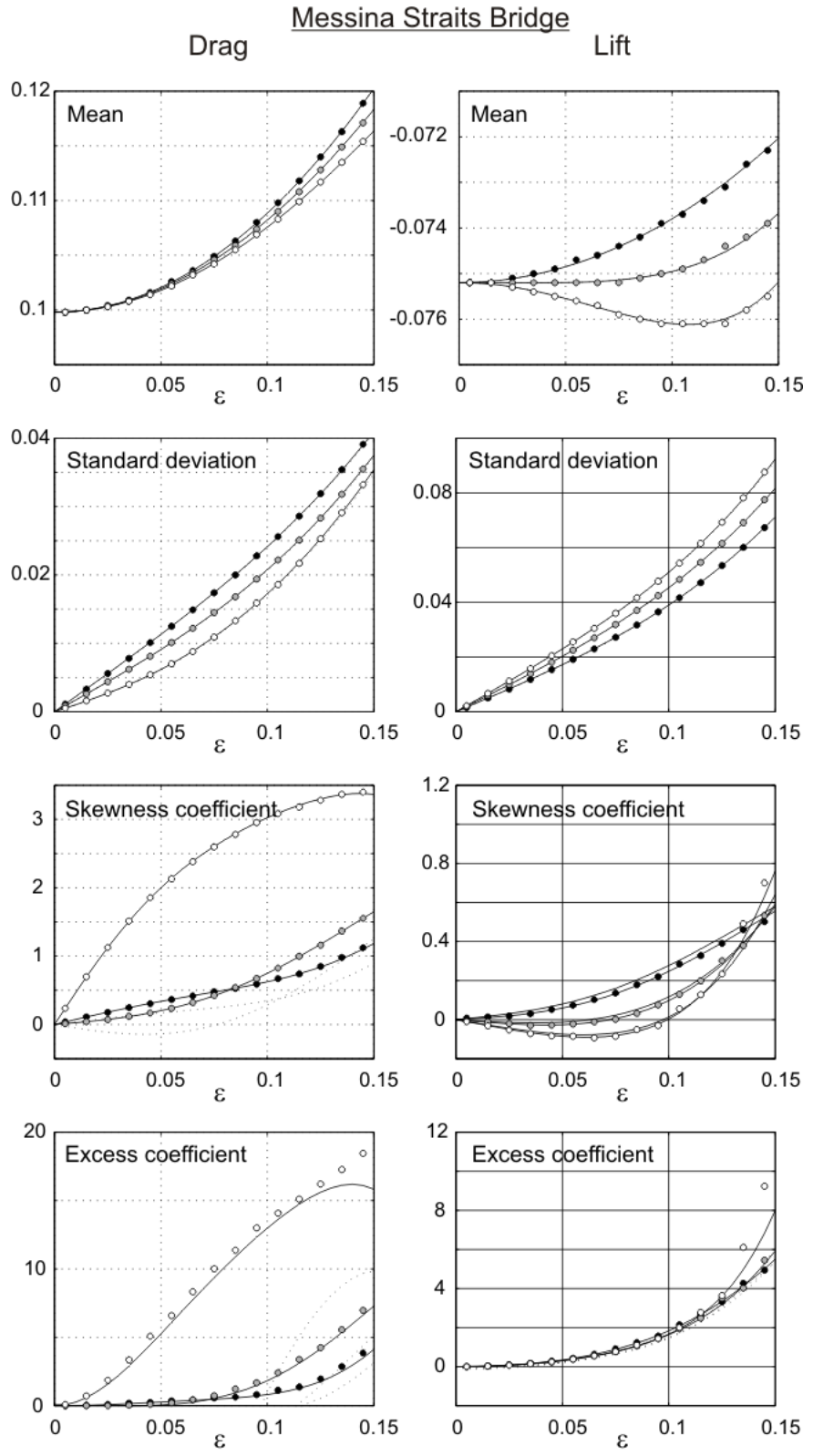

(a)

(b)

\begin{tabular}{|c|c|}
\hline \begin{tabular}{|l} 
Leading +2 corr. \\
$\ldots \ldots$. Leading +1 corr. \\
$0 / 0 / \bullet$ Monte Carlo
\end{tabular} & $\begin{array}{l}-\rho=0 \\
-\rho=-0.5 \\
0 \rho=-1\end{array}$ \\
\hline
\end{tabular}

Figure 4: Statistical characteristics of drag and forces of Messina Straits Bridge $\left(I_{u}=\varepsilon\right.$, $I_{w}=\varepsilon / 2$ ), for various correlation coefficients. Results obtained with the proposed method (lines) compare well with results of Monte Carlo simulations (dots). 

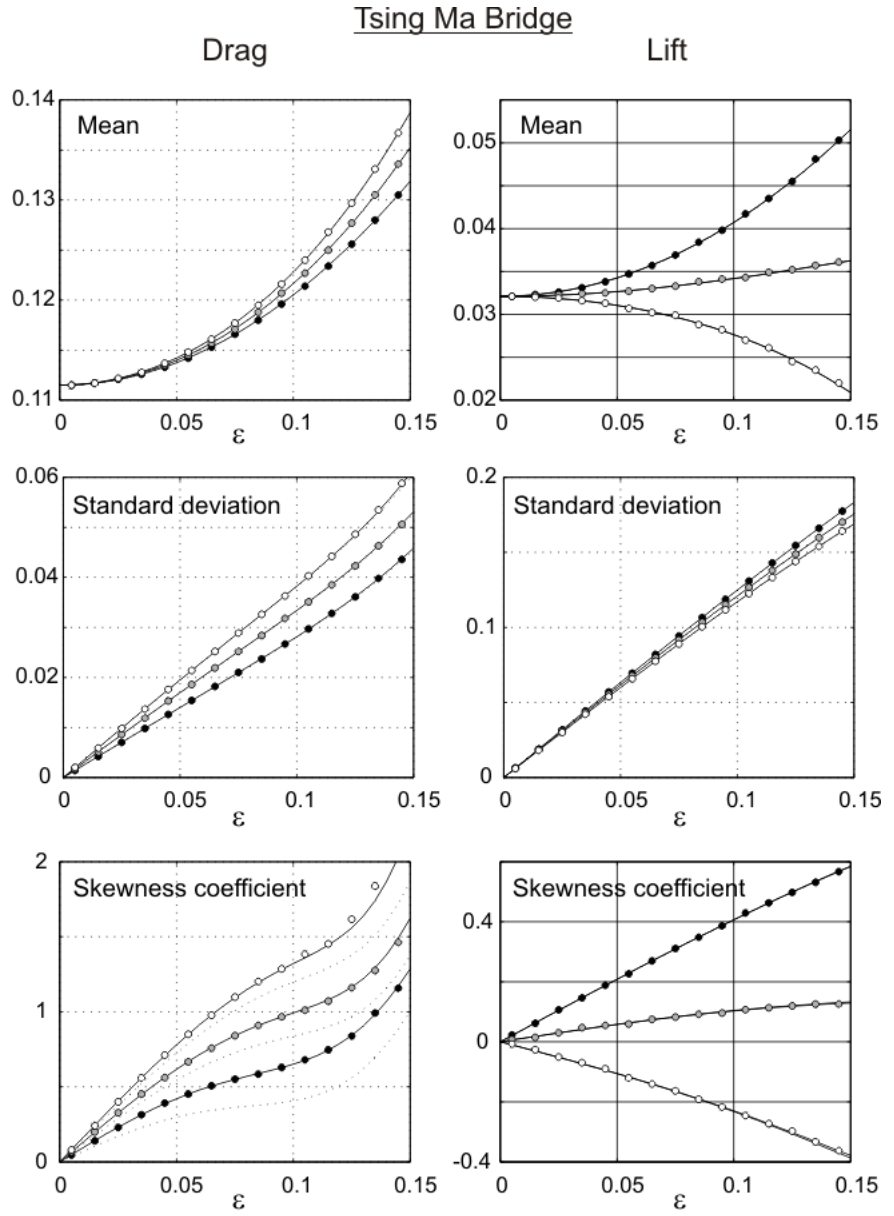

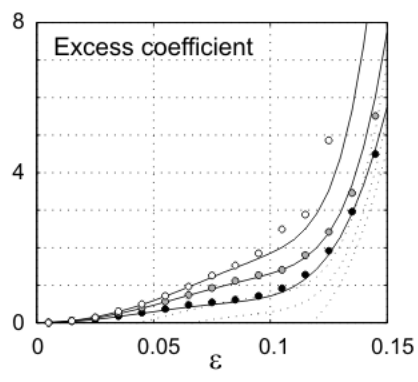

(a)

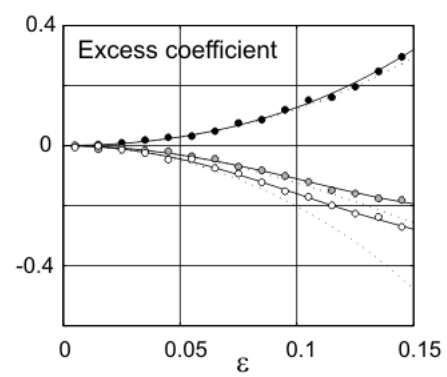

(b)

\begin{tabular}{|c|c|}
\hline $\begin{array}{ll} & \text { Leading }+2 \text { corr. } \\
\cdots \ldots . . & \text { Leading }+1 \text { corr. } \\
\circ / \bullet / \bullet & \text { Monte Carlo }\end{array}$ & $\begin{array}{l}-\rho=0 \\
-\rho=-0.5 \\
-\rho=-1\end{array}$ \\
\hline
\end{tabular}

Figure 5: Statistical characteristics of drag and forces of Tsing Ma Bridge $\left(I_{u}=\varepsilon, I_{w}=\varepsilon / 2\right)$, for various correlation coefficients. Results obtained with the proposed method (lines) compare well with results of Monte Carlo simulations (dots). 


\begin{tabular}{cccc}
\multicolumn{2}{c}{ Messina Straits } & \multicolumn{2}{c}{ Tsing Ma } \\
\hline \hline Drag & Lift & Drag & Lift \\
Best cubic fit & & & \\
$c_{0}=0.0998$ & $c_{0}=-0.0752$ & $c_{0}=0.1115$ & $c_{0}=0.0321$ \\
$c_{1}=0.1942$ & $c_{1}=0.5867$ & $c_{1}=-0.3442$ & $c_{1}=2.5414$ \\
$c_{2}=6.2273$ & $c_{2}=1.8662$ & $c_{2}=6.0894$ & $c_{2}=6.5521$ \\
$c_{3}=-48.3736$ & $c_{3}=93.9708$ & $c_{3}=84.5623$ & $c_{3}=-67.9788$ \\
Best linear fit & & & \\
$c_{0}=0.1219$ & $c_{0}=-0.0686$ & $c_{0}=0.1331$ & $c_{0}=0.0553$ \\
$c_{1}=0.0641$ & $c_{1}=0.8394$ & $c_{1}=-0.1168$ & $c_{1}=2.3586$
\end{tabular}

Table 1: Coefficients of the best linear and cubic fits of drag and lift forces of Messina Straits and Tsing Ma Bridges.

is then performed by establishing histories of the wind incidence and of the squared velocity and multiplying them to yield the history of the aerodynamic force. The statistical properties of the force are finally estimated via unbiased estimators [17]. They are represented by black $(\rho=0)$, gray $(\rho=-0.5)$ and white $(\rho=-1)$ spots in Figs. 4 and 5. The large size of the samples is necessary to provide reliable results for the statistical estimates. It has been chosen here around the limit threshold under which the excess coefficient would not be well identified. This is illustrated by the slight misalignment of spots in plots related to excess coefficients. Because of the need to produce accurate estimates of high order moments, and therefore the need to generate long samples, Monte Carlo simulations are computationally not efficient. Several simulations, for various values of the turbulence intensities, are however performed, independently from each other.

The results of these numerical simulations compare very well with those obtained with the proposed analytical relations, reported as continuous and dashed lines in Figs. 4 and 5 . Continuous lines refer to the leading order solution plus the first correction, whereas results represented by dashed lines include the second correction too. The mean force and the standard deviation virtually coincide with the numerical results, whenever the second correction term is kept or not. Concerning the skewness and excess coefficients, the need to consider the second correction is evident, as dashed lines significantly deviate from continuous ones. For both bridges and both aerodynamic coefficients, the proposed method is able to represent accurately the first four statistical moment. The only criticism that might eventually be formulated concerns the accuracy in the estimation of kurtosis coefficients for large turbulence intensities and a correlation coefficient equal to -1 . Inspection of (45) reveals the reasons for which the proposed expressions provide worse results in case of perfectly correlated turbulence components. Indeed, the presence of a unit correlation coefficient (in absolute value) increases the relative importance of the coefficients of factors in higher powers of $I_{u}$ and $I_{w}$, and therefore cuts down the convergence rate of series (33).

At this stage, we may already point out the large non-Gaussianity of drag forces, compared to lift forces. The major reason for this difference, due to the non-linearity of the aerodynamic force, is illustrated in Section 7.2. The significance of skewness and kurtosis coefficients shows that a Gaussian model of the drag force might provide unacceptable estimates of the loading, especially concerning its extreme values.

Although the correlation coefficient has a limited influence on the mean value and standard 
deviation, it may significantly affect skewness and kurtosis coefficients. This is particularly exacerbated when the aerodynamic coefficient manifests a significant non-linearity, i.e. for drag coefficients in these applications. Furthermore, it should be noted that statistical moments are not expressed as monotonic functions of the correlation coefficient, as is indicated by the crossing of two curves related to the skewness of the drag force of Messina Straits Bridge (Fig. 4-a). Also the correlation coefficient may, in some cases, influence significantly the skewness coefficient. For instance, the lift force on Tsing Ma Bridge is positively skewed for uncorrelated turbulence components $(\rho=0)$, whereas it is negatively skewed for perfectly correlated turbulence components $(\rho=-1)$.

The outstanding ability of the proposed method to fit the exact simulation results is quite impressive. This illustrates the potential of perturbation methods and is such here essentially because several limit analyses are performed independently for each coefficient $c_{i}$ or combination of coefficients $c_{i}$ 's. Thanks to this particularity of the model, results obtained with the proposed relations are able to follow precisely the succession of curvature alternations of the plots of Figs. 4 and 5, in spite of the application of a limit analysis. Of course, this would not have been so if a crude Taylor series expansion, limited to the leading order plus a couple of corrections, of the statistical characteristics of interest had been performed. Indeed, in this case, continuous and dashed lines of Figs. 4 and 5 would exhibit quadratic or cubic (at most) forms. In other words, thanks to the fact that the limit analysis is performed separately on each product of $c_{i}^{\prime} s$, the proposed relations provide precise high-order approximations of the statistical characteristics of the loading.

\subsection{Comparison with traditional models}

In this section, we compare the non-linear loading model with two more traditional models similar to those introduced in Section 1, but in a two-dimensional wind flow. In order to assure a comparison of the models and not of analytical expressions of the corresponding statistical moments (eventually approximate), the comparison between the three models is performed through Monte Carlo simulations. Thus, similarly to the previous application, series of wind histories are generated for each model, using (10) for the non-linear model and (50) and (53) that are next developed for the two traditional models. Statistical characteristics of the different loadings are then estimated via unbiased estimators. This numerical simulation is performed under similar conditions concerning the turbulence intensities, but is now limited to $\rho=-0.5$. Results of the non-linear model, symbolized by gray dots in Figs. (4) and (5), are reported in Figs. (6) and (7) in order to allow a better comparison with the two traditional models.

First, the most usual linear model is considered [3], for which the expression of the dimensionless force is obtained from the first terms of the Taylor series expansion of (10),

$$
f=c_{0}\left(1+\frac{2 u}{U}\right)+c_{1} w
$$

Although analytical relations are not used in this section, it is immediate to obtain explicit 

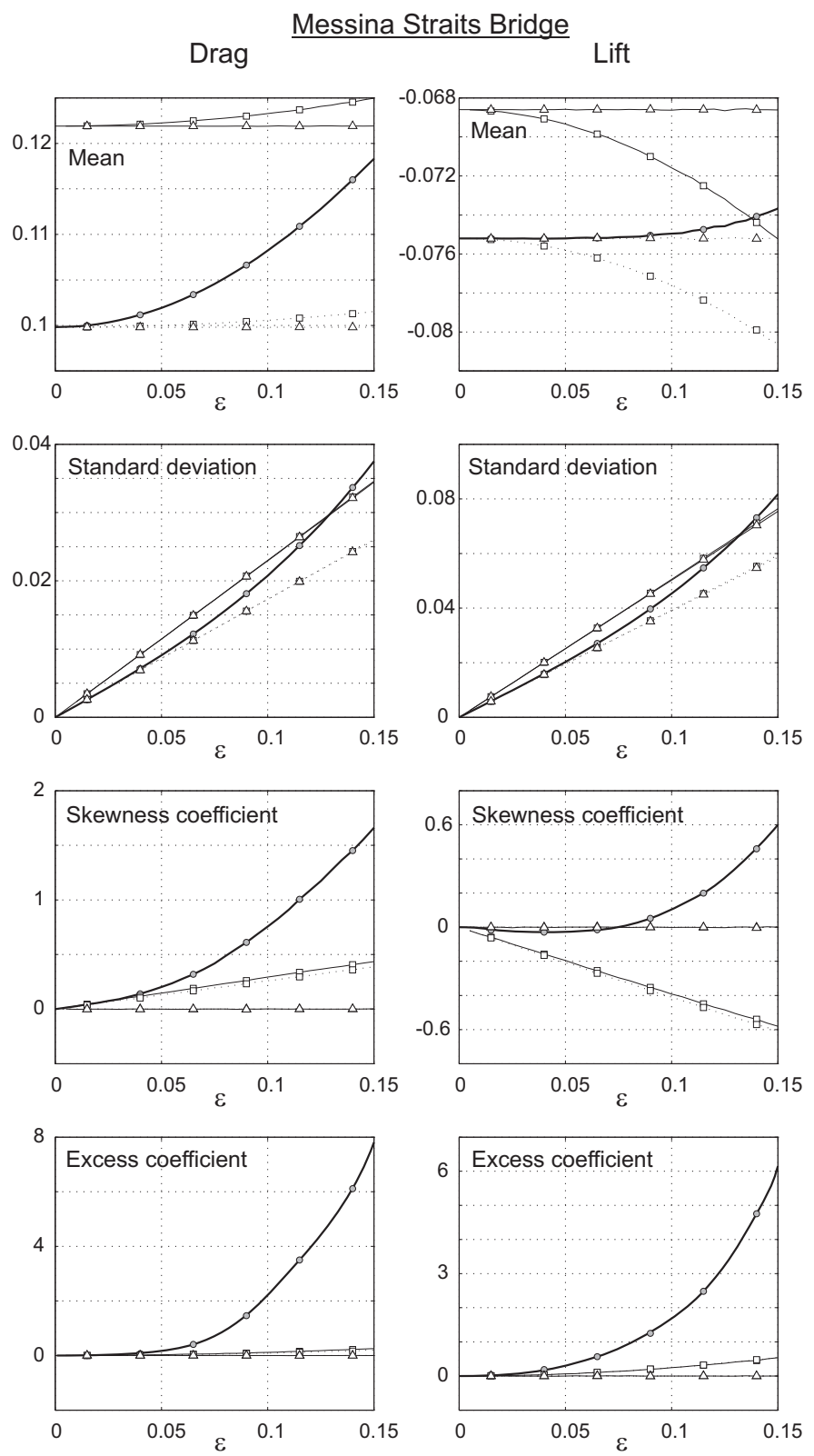

(a)

(b)

$\square-$ Non-linear model
$\cdots \quad$ Quad w. linear coef (with $c_{0}, c_{1}$ from best cubic fit)
$\square-$ Quad w. linear coef (with $c_{0}, c_{1}$ from best linear fit)
$\square-$ Linear model (with $c_{0}, c_{1}$ from best cubic fit)
$\square-$ Linear model (with $c_{0}, c_{1}$ from best linear fit)

Figure 6: Comparison of statistical characteristics of drag and lift forces of Messina Bridge $\left(I_{u}=\varepsilon, I_{w}=\varepsilon / 2, \rho=-0.5\right)$ for various models. Gray dots (non-linear model) are identical to those of Fig. 4. Triangles and squares represent the results obtained with the linear model and the quadratic model (however limited to linear aerodynamic coefficients), respectively. 

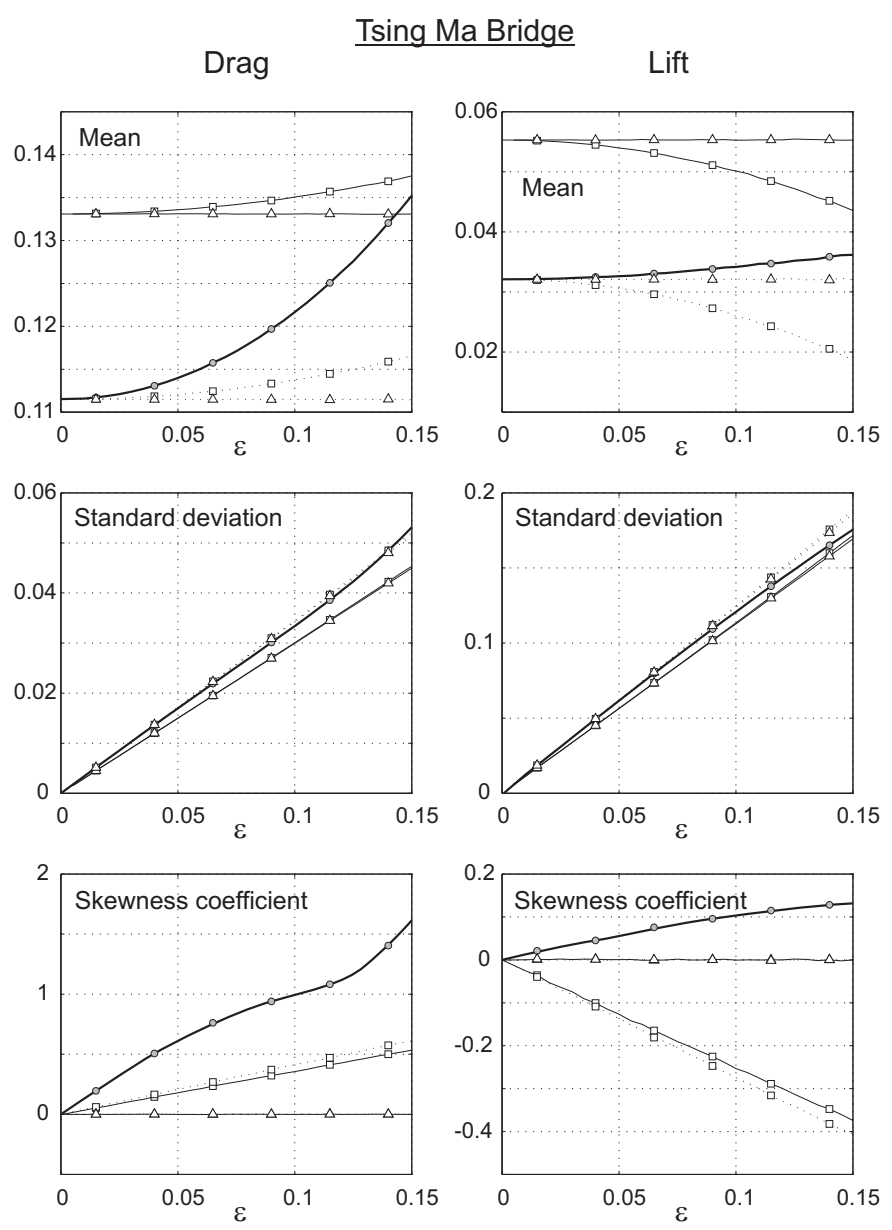

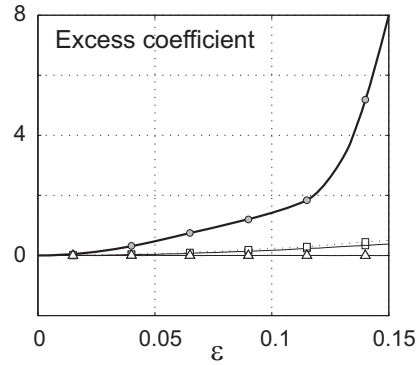

(a)

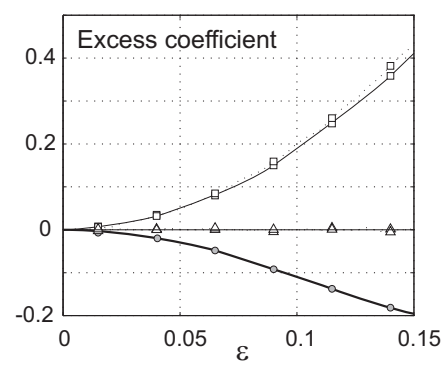

(b)

- Non-linear model
$\cdots \quad$ Quad w. linear coef (with $c_{0}, c_{1}$ from best cubic fit)
$\square$ Quad w. linear coef (with $c_{0}, c_{1}$ from best linear fit)
$\therefore-$ Linear model (with $c_{0}, c_{1}$ from best cubic fit)
$\square-$ Linear model (with $c_{0}, c_{1}$ from best linear fit)

Figure 7: Comparison of statistical characteristics of drag and lift forces of Tsing Ma Bridge $\left(I_{u}=\varepsilon, I_{w}=\varepsilon / 2, \rho=-0.5\right)$ for various models. Gray dots (non-linear model) are identical to those of Fig. 5. Triangles and squares represent the results obtained with the linear model and the quadratic model (however limited to linear aerodynamic coefficients), respectively. 
relations for the first statistical characteristics

$$
\begin{aligned}
\mu_{f} & =c_{0} \\
\mu_{f^{2}} & =c_{0}^{2}\left(1+4 I_{u}^{2}\right)+c_{1}^{2} I_{w}^{2}+4 c_{0} c_{1} \rho I_{u} I_{w} \\
\kappa_{f^{3}} & =0 \\
\kappa_{f^{4}} & =0 .
\end{aligned}
$$

They confirm the well-known property stating that a linear transformation of a multivariate Gaussian variable remains Gaussian. Comparison of (51) to (30) and (44) shows that this linear model and the non-linear model considered in this paper both degenerate to identical values for low turbulence intensities. The statistical characteristics of this loading are represented by triangles in Figs. (6) and (7). Two results are presented in each plot: continuous lines refer to the characteristics obtained with the values of $c_{0}$ and $c_{1}$ corresponding to the best linear fit, see Table 1, whereas dashed lines refer to the linear model obtained with the first two coefficients of the best cubic fit. This latter representation illustrates the degenerescence of the linear and non-linear models to the same limits, but has not much interest. Indeed this linear representation of the aerodynamic coefficient corresponds roughly to the tangent to the measured data for a zero wind incidence (see Fig. 2), and is therefore valid for small intensities only. On the contrary, the continuous lines -referring to the best linear fit- allow a better representation of the aerodynamic coefficient, along the whole domain of wind incidence. This justifies the better matching with results of the non-linear fit, despite the fact that the behaviors are not asymptotically convergent.

Whichever set of coefficients is considered, Monte Carlo simulations show that the mean force is constant and that skewness and kurtosis coefficients are null, which is in agreement with the explicit expressions (51). In the linear model, the standard deviation is proportional to the turbulence intensities as might be observed in Figs. (6) and (7), but also from (51)

$$
\sigma_{f}=\sqrt{\mu_{f^{2}}-\mu_{f}^{2}}=\varepsilon \sqrt{4 c_{0}^{2}-c_{0} c_{1}+c_{1}^{2} / 4}
$$

which is obtained by considering $I_{u}=\varepsilon, I_{w}=\varepsilon / 2$ and $\rho=-0.5$.

For the considered example, the discrepancy on the standard deviation remains usually within acceptable limits, showing however a 15\%-offset for the drag coefficient of the Tsing Ma Bridge in case of large turbulence intensities. The most serious issue concerning the use of a linear model consists in a failing estimation of higher order moments, and therefore of the peak factor, which consequently leaves the discrepancy on the mean force of marginal importance.

Secondly, we consider a non-linear model accounting for the quadratic form of the wind velocity but limited to a linear representation of the aerodynamic coefficient. It is an extension, in a $2-\mathrm{D}$ wind field, of the work presented in $[8,9,10,11]$. The dimensionless aerodynamic force writes

$$
f=c_{0}\left(1+\frac{2 u}{U}+\frac{u^{2}}{U^{2}}+\frac{w^{2}}{U^{2}}\right)+c_{1} \frac{w}{U}\left(1+\frac{u}{U}\right) .
$$

In view of this formulation, this model is referred to as quadratic loading in the following. Again, despite the fact that the problem is illustrated with Monte Carlo simulations, it is interesting to develop analytical expressions of the statistical moments of this loading. After some developments and simplifications, one may obtain 


$$
\begin{aligned}
\mu_{f}= & c_{0}\left(1+I_{u}^{2}+I_{w}^{2}\right)+I_{u} I_{w} \rho c_{1} \\
\mu_{f^{2}}= & c_{0}^{2}\left(1+6 I_{u}^{2}+2 I_{w}^{2}+5 I_{u}^{4}+3 I_{w}^{4}+4 \rho^{2} I_{u}^{2} I_{w}^{2}\right)+c_{1}^{2} I_{w}^{2}\left(1+\left(1+2 \rho^{2}\right) I_{u}^{2}\right)+ \\
& +6 c_{0} c_{1} I_{u} I_{w} \rho\left(1+I_{u}^{2}+I_{w}^{2}\right)
\end{aligned}
$$

for the first two moments. Expressions of the third and fourth moments are not provided because of an excessive clumsiness. These relations are not straightforwardly obtained by setting $c_{2}=0$ and $c_{3}=0$ in (30) and (44), especially concerning the second moment. The first reason is due to the exactness of (54), opposed to the approximate character of (44). This justifies the existence of higher order terms (as $5 I_{u}^{4} c_{0}^{2}$ ) in (54) which are evidently negligible, in view of the arguments presented in this paper. The second reason is due to the consideration of the non linear geometric expression of the wind incidence (11) in the complete model, which justifies by contrast the difference in some coefficients of similar terms, e. g. $c_{0} c_{1} I_{u} I_{w}^{3} \rho$. These differences are so minor that the disagreement between the quadratic model and the non-linear one is mostly attributable to the non-linearity of the aerodynamic coefficient, i.e. non-zero values of $c_{2}$ and $c_{3}$.

The statistical characteristics resulting from this particular quadratic loading are represented by squares in Figs. (6) and (7), and again continuous and dashed lines refer to different selections of $c_{0}$ and $c_{1}$. In general, the consideration of the quadratic model provides a quadratic representation of the mean force, which is however not curved enough or curved in the wrong direction to match the mean force obtained with the non-linear model. This is not further discussed as the discrepancy on the estimation of the mean force is marginal. Standard deviations obtained with the quadratic model virtually match those resulting from the linear model, which confirms the main conclusions presented in $[8,9,10,11]$. Actually in these pioneering works, the need to consider a quadratic loading was mainly motivated by the non-Gaussianity of the loading, and not the influence on the standard deviation. Indeed, at that time, only comparison with the linear model was possible, and only the significant values of the skewness and kurtosis coefficients could be evoked to promote the use of a quadratic model. Now, comparison with a more general non-linear model is possible, and indicates that the neglection of the non-linearity of aerodynamic coefficients may lead to imprecise representation of the non-Gaussian loading. At least, if the non-Gaussianity of the quadratic model is accepted, no questioning should be considered for the non-linear model.

\section{Conclusions}

Based on a non-linear quasi-steady wind loading, accounting for the squared velocity, the nonlinear geometric expression of the wind incidence and more notably non-linear aerodynamic coefficients, we have proposed to construct approximate expressions of the raw moments of aerodynamic forces by means of a dedicated limit analysis. This analysis is performed independently for different coefficients in order to present an acceptable global accuracy, regardless of the shape of the non-linear aerodynamic coefficients. In the approximate expressions of the raw moments, the leading order as well as the first two corrections are given. With aerodynamic coefficients and wind intensities encountered in practical applications, the leading order term alone gives a very good estimate of the raw moments. However, as demonstrated with a

counterexample, cumulants and other dimensionless quantities, such as skewness and excess 
coefficients, require the first two corrections, owing to the cancellation of terms of identical orders.

Based on Monte Carlo simulations, a comparison of the expressions resulting from the proposed limit analysis indicates a high accuracy for turbulence intensities as large as $I_{u}=$ $10 \%$, depending on the correlation coefficient between both turbulence components. It is evident that the computation effort necessary to the use of the proposed relation is insignificant in contrast with that required for Monte Carlo simulations. This make therefore the proposed relations an appropriate tool for parametric studies.

Finally the results of the considered non-linear model are compared to those of more traditional models as the linear and quadratic (with linear coefficients) models. Basically the difference between the two traditional models are limited to non-zero values of skewness and excess coefficients in the latter, as opposed to zero values for the Gaussian processes of the linear model. With the consideration of the non-linear model, these observations are amplified: the mean force and standard deviation of the force may significantly differ from the linear model to the non-linear one, and skewness and excess coefficients take even larger values.

As a conclusion, the developments presented in this paper suggest the need to consider higher order statistical characteristics of the quasi-steady aerodynamic loading, especially in case of non-linear aerodynamic coefficients.

\section{Acknowledgments}

First, we would like to acknowledge the Belgian Fund for Scientific Research for having provided the financial means to perform this research. The brilliant idea of considering the smallness of some parameters arose from a fruitful stay at the Commonwealth Scientific and Industrial Research Organization, Australia (2007-2008), in a division headed by Prof. E. Detournay. Although the considered applications of our common research were completely different, the developments presented in this paper were inspired by the physical intuition and stunning sense of modeling that is fostered in his team. The motivation brought by the early mathematical developments goes to Yevhen Kovalyshen, University of Minnesota. Special thanks also go to Ir. C. Braham for the criticism that helped improve markedly the paper's final form.

\section{References}

[1] C. Dyrbye and S. O. Hansen. Wind loads on structures. 1997.

[2] J. G. Jones. Measured statistics of multicomponent gust patterns in atmospheric turbulence. Journal Of Aircraft, 44:1559-1567, 2007. Times Cited: 0.

[3] E. Simiu and R. Scanlan. Wind Effects On Structures. John Wiley and Sons, 3rd edition, 1996.

[4] EN 1991-1-4. Eurocode 1: Actions on structures part 1-4: General actions: Wind actions. 1991. 
[5] V. Denoël. Polynomial approximation of aerodynamic coefficients based on the statistical description of the wind incidence. Probabilistic Engineering Mechanics, In Press, Accepted Manuscript.

[6] X. Z. Chen and A. Kareem. Advances in modeling of aerodynamic forces on bridge decks. Journal Of Engineering Mechanics-Asce, 128(11):1193-1205, 2002. Times Cited: 13.

[7] H. W. Liepmann. On the application of statistical concepts to the buffeting problem. Journal of Aeronautical Sciences, 19(12):793-800, 1952.

[8] L. D. Lutes and S. L. J. Hu. Nonnormal stochastic response of linear-systems. Journal Of Engineering Mechanics-Asce, 112(2):127-141, 1986. Times Cited: 29.

[9] G. Muscolino. Linear-systems excited by polynomial forms of non-gaussian filtered processes. Probabilistic Engineering Mechanics, 10(1):35-44, 1995. Times Cited: 23.

[10] S. Benfratello, M. Di Paola, and P. D. Spanos. Stochastic response of mdof wind-excited structures by means of volterra series approach. Journal Of Wind Engineering And Industrial Aerodynamics, 74-6:1135-1145, 1998. Times Cited: 7.

[11] V. Gusella and A. L. Materazzi. Non-gaussian along-wind response analysis in time and frequency domains. Engineering Structures, 22(1):49-57, 2000. Times Cited: 3.

[12] V. Denoël and H. Degée. Influence of the non-linearity of the aerodynamic coefficients on the skewness of the buffeting drag force. Wind And Structures, 9(6):457-471, 2006. Times Cited: 0.

[13] G. Diana, M. Falco, F. Cheli, and A. Cigada. The aeroelastic study of the messina straits bridge. Natural Hazards, 30(1):79-106, 2003. Times Cited: 0.

[14] L. D. Zhu, Y. L. Xu, F. Zhang, and H. F. Xiang. Tsing ma bridge deck under skew winds - part i: Aerodynamic coefficients. Journal Of Wind Engineering And Industrial Aerodynamics, 90(7):781-805, 2002. Times Cited: 6.

[15] M. Abramowitz and I. A. Stegun. Handbook of Mathematical Functions with Formulas, Graphs, and Mathematical Tables. New-York, 1972.

[16] G. Solari and G. Piccardo. Probabilistic 3-d turbulence modeling for gust buffeting of structures. Probabilistic Engineering Mechanics, 16(1):73-86, 2001. doi: DOI: 10.1016/S0266-8920(00)00010-2.

[17] A. Papoulis. Probability, Random Variables, and Stochastic Processes. McGraw Hill, New York, 1965. 